\title{
Association between chronic kidney disease and COVID-19-related mortality in New York
}

\author{
Nihal E. Mohamed ${ }^{1} \cdot$ Emma K. T. Benn $^{2} \cdot$ Varuna Astha $^{2} \cdot$ Kennedy E. Okhawere ${ }^{1} \cdot$ Talia G. Korn $^{1} \cdot$ \\ William Nkemdirim ${ }^{1}$ - Ami Rambhia ${ }^{1}$. Olajumoke A. Ige ${ }^{1}$. Hassan Funchess ${ }^{1} \cdot$ Meredith Mihalopoulos $^{1}$. \\ Kirolos N. Meilika ${ }^{1} \cdot$ Natasha Kyprianou ${ }^{1,3,4} \cdot$ Ketan K. Badani ${ }^{1,3}$ (1)
}

Received: 14 August 2020 / Accepted: 12 December 2020 / Published online: 22 January 2021

(c) The Author(s), under exclusive licence to Springer-Verlag GmbH, DE part of Springer Nature 2021

\begin{abstract}
Purpose To evaluate mortality risk of CKD patients infected with COVID-19, and assess shared characteristics associated with health disparities in CKD outcome.

Methods We extracted the data from a case series of 7624 patients presented at Mount Sinai Health System, in New York for testing between 3/28/2020 and 4/16/2020. De-identified patient data set is being produced by the Scientific Computing department and made available to the Mount Sinai research community at the following website: https://msdw.mountsinai.org/. Results Of 7624 COVID-19 patients, 7.8\% ( $n=597)$ had CKD on hospital admission, and 11.2\% ( $n=856)$ died of COVID-19 infection. CKD patients were older, more likely to have diabetes, hypertension, and chronic obstructive pulmonary disease (COPD), were current or former smokers, had a longer time to discharge, and had worse survival compared to non-CKD patients $(p<0.05)$. COVID-19 mortality rate was significantly higher in CKD patients $(23.1 \%$ vs $10.2 \%)$ with a 1.51 greater odds of dying (95\% CI: 1.19-1.90). Controlling for demographic, behavioral, and clinical covariates, the logistic regression analysis showed significant and consistent effects of CKD, older age, male gender, and hypertension with mortality $(p<0.05)$. Conclusion CKD was a significant independent predictor of COVID-19 mortality, along with older age, male gender, and hypertension. Future research will investigate the effects of COVID-19 on long-term renal function.
\end{abstract}

Keywords COVID- $19 \cdot$ Chronic kidney disease $\cdot$ Comorbidity $\cdot$ Mortality $\cdot$ Virus $\cdot$ Pandemic

Nihal E. Mohamed and Emma K. T. Benn are first co-authors.

Ketan K. Badani

ketan.badani@mountsinai.org

1 Department of Urology, Icahn School of Medicine at Mount Sinai, 6th Floor, 1425, Madison Ave, New York, NY 10029, USA

2 Department of Population Health Science and Policy, Center for Biostatistics, Icahn School of Medicine at Mount Sinai, New York, NY 10029, USA

3 Tisch Cancer Institute at Mount Sinai, New York, NY 10029, USA

4 Department of Oncological Sciences, Icahn School of Medicine at Mount Sinai, New York, NY 10029, USA

\section{Introduction}

The World Health Organization (WHO) deemed COVID19 disease (nCov-2019) a global pandemic on March 11th, 2020 [1]. Of the 856,209 reported cases in the United States (US), 47,272 resulted in mortality, leading to a case fatality of approximately 18\% as of April 23rd, 2020 [2].

The first case of COVID-19 was reported on December 31, 2019 in Wuhan, China, and since then, the virus has spread exponentially around the world [3]. A growing number of COVID-19 cases have emerged in New York, deeming it the epicenter of the COVID-19 outbreak in the United States. There are over 257,200 reported cases and 15,300 deaths in New York as of April 22nd, 2020, accounting for nearly $32 \%$ of COVID-19 deaths in US [4, 5].

Globally, factors such as older age, and patient pre-existing health conditions have been associated with the case fatality rate (CFR). Data from the China Center of Disease Control (CDC) show that patients with diabetes mellitus, 
obesity, chronic lung disease, heart, liver, and chronic kidney disease (CKD) also have a higher CFR [6]. As of April 22, 2020 , the CFR of the world is $7 \%$ overall, but has fluctuated throughout the outbreak [7].

Previous studies have shown that patients with CKD with comorbidities have poorer outcomes such as all-cause mortality, increased hospitalization, and longer hospital stays than comparative individuals [8]. Additionally, there is evidence that suggests racial disparities in CKD mortality [9]. Emerging data indicate that patients with poor kidney function also experience severe COVID-19-related outcomes [10].

In this study, we examine the role of CKD in COVID19-related mortality, as well as, evidence of shared demographic and clinical characteristics associated with documented health disparities.

\section{Materials and methods}

Our study consists of the largest US case series of patients diagnosed with COVID-19 across the Mount Sinai Health System (MSHS), in New York, from March 28th, 2020 to April 16th, 2020. As MSDW provides de-identified data, the Ethics Committee of MSHS approved a waiver of documentation of informed consent. Data were collected on COVID19 patients that were admitted to the hospital according to Mount Sinai Health System guidelines [11]. Only patients that were discharged were included in the analysis. Respiratory specimens were collected by the medical staff in all affiliated hospitals and then shipped to designated MSHS authorized laboratories to detect COVID-19. The presence of SARS-CoV-2 in respiratory specimens was detected by real-time RT-PCR methods. The RT-PCR assay was conducted in accordance with the protocol established by the WHO [12]. De-identified COVID-19 patient data sets are made available daily by the Scientific Computing department to the Mount Sinai research community at the Mount Sinai Data Warehouse (MSDW) [https://msdw.mountsinai .org/]. These data included information on patient demographics, comorbidities, body-mass index, smoking status, date of diagnosis, date of hospitalization, date of discharge, or date of death for patients with a COVID-19-related encounter.

Data were summarized as median (interquartile range) for continuous variables and as frequency (\%) for categorical variables. We described the distribution of demographics, smoking status, comorbidities, and outcomes among COVID-19-positive patients overall and stratified by CKD status. CKD stage was defined using the Modification of Diet in Renal Disease (MDRD) as estimated GFR (eGFR) less than $60 \mathrm{~mL} / \mathrm{min} / 1.73 \mathrm{~m}^{2}$, according to National Kidney Foundation Practice Guidelines. [13, 14]. Bivariate tests examined associations between the demographic (age, race/ ethnicity, sex), behavioral (smoking history, obesity), and clinical variables (i.e., comorbidities) as well as mortality, discharge, time to death or discharge and CKD (stage 3-5) status (yes/no) using Chi-square $\left(\chi^{2}\right)$ or Fisher's exact tests for categorical variables and Wilcoxon rank sum tests or Kruskal-Wallis tests for continuous variables. Subsequently, we evaluated the associations between these variables and mortality (yes/no). In a series of logistic regression analyses, we examined the relationship between CKD and mortality controlling for potential demographics, behavioral, and clinical covariates. Only variables significantly associated with mortality in bivariate assessments at the $\alpha=0.10$ level were included in our logistic regression models. Statistical analyses were performed using SAS 9.4 and R 3.6.

\section{Results}

Of the 7624 COVID-19 patients in our sample, $7.8 \%$ $(n=597)$ had a diagnosis of CKD (Table 1). Approximately $32.8 \%$ of patients were at least 65 years of age. The majority $(54.6 \%)$ of the patients were male and almost half of the patients were of African diasporic ancestry (24.9\%) or Hispanic/Latinx ancestry (24.4\%). About one-fifth (20.4\%) of patients were current or former smokers, and $6.7 \%$ were obese. With respect to comorbidities, $4.4 \%$ of patients presented with asthma, $2.6 \%$ with chronic obstructive pulmonary (COPD), $24.7 \%$ with hypertension, $17.6 \%$ with diabetes, $1.6 \%$ with human immunodeficiency viruses (HIV), and $6.3 \%$ with cancer. More than one-tenth (11.2\%) of patients died during the observation period of this study. Among those who did not die, $51.6 \%$ were discharged, $10.0 \%$ were outpatients, and $27.1 \%$ remained hospitalized at the time of this analysis.

We observed statistically significant differences between patients with and without CKD with respect to age, race/ ethnicity, smoking status, obesity, all comorbidities, and mortality ( $p<0.001$; Table 1$)$. More specifically, patients with CKD were older (62.8\%) compared to the non-CKD group $(36.1 \%)$. About two-thirds of patients with CKD were of African diasporic ancestry (34.3\%) or Hispanic/Latinx (34.5\%) compared to $24.1 \%$, and $23.6 \%$, respectively, in the non-CKD group. The prevalence of being a current or former smoker was more than twofold higher in the CKD group (41.0\%) compared to the non-CKD group (18.7\%), while the prevalence of obesity was about threefold higher $(17.9 \%$ vs 5.7\%). Asthma and COPD were prevalent among $8.9 \%$, and $7.7 \%$ of the CKD group, respectively. We observed fourfold higher prevalence of hypertension $(80.4 \%$ vs $20.0 \%)$ and diabetes (56.4\% vs $14.3 \%$ ) in patients with CKD. The prevalence of HIV and cancer in CKD patients was $4.0 \%$ and $5.9 \%$, respectively. Almost one-quarter (23.1\%) of those 
Table 1 Distribution of demographics, smoking status, comorbidities, and outcomes among COVID-19-positive patients overall and stratified by chronic kidney disease

\begin{tabular}{|c|c|c|c|c|c|}
\hline Variable & Sub-categories & Overall $(n=7624)$ & $\begin{array}{l}\text { Non-CKD } \\
\text { patients }(n=7027 \text {, } \\
92.2 \%)\end{array}$ & $\begin{array}{l}\text { CKD patients } \\
(n=597 \\
7.8 \%)\end{array}$ & $p$ value \\
\hline \multirow[t]{3}{*}{ Age } & $<45$ years & $2093(27.4 \%)$ & $2048(29.1 \%)$ & $45(7.5 \%)$ & \multirow[t]{3}{*}{$<0.001$} \\
\hline & 45 to $<65$ years & $2621(34.4 \%)$ & $2444(34.8 \%)$ & $177(29.6 \%)$ & \\
\hline & $65+$ years & $2910(38.2 \%)$ & $2535(36.1 \%)$ & $375(62.8 \%)$ & \\
\hline \multirow[t]{2}{*}{$\operatorname{Sex}^{\mathrm{a}}$} & Female & $3450(45.2 \%)$ & $3190(45.4 \%)$ & $260(43.6 \%)$ & \multirow[t]{2}{*}{0.38} \\
\hline & Male & $4161(54.6 \%)$ & $3824(54.4 \%)$ & $337(56.4 \%)$ & \\
\hline \multirow[t]{5}{*}{ Race/ethnicity } & African ancestry & $1898(24.9 \%)$ & $1693(24.1 \%)$ & $205(34.3 \%)$ & \multirow[t]{5}{*}{$<0.001$} \\
\hline & White & $1894(24.8 \%)$ & $1805(25.7 \%)$ & $89(14.9 \%)$ & \\
\hline & Hispanic/Latinx & $1862(24.4 \%)$ & $1656(23.6 \%)$ & $206(34.5 \%)$ & \\
\hline & Asian & $357(4.7 \%)$ & $332(4.7 \%)$ & $25(4.2 \%)$ & \\
\hline & Other/unknown & $1613(21.2 \%)$ & $1541(21.9 \%)$ & $72(12.1 \%)$ & \\
\hline \multirow[t]{3}{*}{ Smoking status } & Non-smoker & $3906(51.2 \%)$ & $3581(51.0 \%)$ & $325(54.4 \%)$ & \multirow[t]{3}{*}{$<0.001$} \\
\hline & Current/former smoker & $1558(20.4 \%)$ & $1313(18.7 \%)$ & $245(41.0 \%)$ & \\
\hline & Unknown & $2160(28.3 \%)$ & $2133(30.3 \%)$ & $27(4.5 \%)$ & \\
\hline \multirow[t]{2}{*}{ Asthma } & No & $7289(95.6 \%)$ & $6745(96.0 \%)$ & $544(91.1 \%)$ & \multirow[t]{2}{*}{$<0.001$} \\
\hline & Yes & $335(4.4 \%)$ & $282(4.0 \%)$ & $53(8.9 \%)$ & \\
\hline \multirow[t]{2}{*}{ COPD } & No & $7426(97.4 \%)$ & $6875(97.8 \%)$ & $551(92.3 \%)$ & \multirow[t]{2}{*}{$<0.001$} \\
\hline & Yes & $198(2.6 \%)$ & $152(2.2 \%)$ & $46(7.7 \%)$ & \\
\hline \multirow[t]{2}{*}{ HTN } & No & $5737(75.2 \%)$ & $5620(80.0 \%)$ & $117(19.6 \%)$ & \multirow[t]{2}{*}{$<0.001$} \\
\hline & Yes & $1887(24.7 \%)$ & $1407(20.0 \%)$ & $480(80.4 \%)$ & \\
\hline \multirow[t]{2}{*}{ Obesity } & No & $7114(93.3 \%)$ & $6624(94.3 \%)$ & $490(82.1 \%)$ & \multirow[t]{2}{*}{$<0.001$} \\
\hline & Yes & $510(6.7 \%)$ & $403(5.7 \%)$ & $107(17.9 \%)$ & \\
\hline \multirow[t]{2}{*}{ Diabetes } & No & $6280(82.4 \%)$ & $6020(85.7 \%)$ & $260(43.6 \%)$ & \multirow[t]{2}{*}{$<0.001$} \\
\hline & Yes & $1344(17.6 \%)$ & $1007(14.3 \%)$ & $337(56.4 \%)$ & \\
\hline \multirow[t]{2}{*}{ HIV } & No & $7503(98.4 \%)$ & $6930(98.6 \%)$ & $573(96.0 \%)$ & \multirow[t]{2}{*}{$<0.001$} \\
\hline & Yes & $121(1.6 \%)$ & $97(1.4 \%)$ & $24(4.0 \%)$ & \\
\hline \multirow[t]{2}{*}{ Cancer } & No & $7140(93.6 \%)$ & $6613(94.1 \%)$ & $527(88.3 \%)$ & \multirow[t]{2}{*}{$<0.001$} \\
\hline & Yes & $484(6.3 \%)$ & $414(5.9 \%)$ & $70(11.7 \%)$ & \\
\hline \multirow[t]{2}{*}{ Death } & No & $6768(88.7 \%)$ & $6309(89.8 \%)$ & $459(76.9 \%)$ & \multirow[t]{2}{*}{$<0.001$} \\
\hline & Yes & $856(11.2 \%)$ & $718(10.2 \%)$ & $138(23.1 \%)$ & \\
\hline
\end{tabular}

Data were summarized as median (IQR) for continuous variables and as frequency (column \%) for categorical variables

${ }^{\mathrm{a}}$ Missing information on sex for $13(0.2 \%)$ overall, all of which pertained to the non-CKD group with CKD died during the study observation period as compared to about one-tenth $(10.2 \%)$ of patients in the non-CKD group.

All variables under consideration were associated with mortality at the $\alpha=0.10$ level except asthma ( $p=0.41)$, obesity $(p=0.33)$, and HIV ( $p=0.30$; Table 2$)$. Almost onequarter $(22.6 \%)$ of patients aged 65 years and older died as compared to $6.9 \%$ and $0.9 \%$ among 45 to $<65$ year olds and $<45$ year olds, respectively $(p<0.001)$. Approximately $12.1 \%$ of males died compared with $10.2 \%$ among females $(p=0.011)$. Slightly higher proportions of those of African diasporic ancestry (11.9\%) and Whites (12.7\%) died compared to Hispanic/Latinx (10.1\%), Asian (10.9\%), and Other/ Unknown $(10.0 \%)$ racial/ethnic groups $(p=0.054)$. Approximately $16.1 \%$ of current/former smokers died compared to $10.4 \%$ of non-smokers and $9.2 \%$ of those with unknown smoking status $(p<0.001)$. Approximately $23.7 \%$ of patients with COPD died compared to $10.9 \%$ of those without COPD $(p<0.001)$. Compared to those without hypertension, more patients with hypertension died $(8.7 \%$ vs $19.0 \%, p<0.001)$. Similarly, more deaths were observed among patients with diabetes $(18.0 \%$ vs $9.8 \%, p<0.001)$ and cancer $(15.3 \%$ vs $10.9 \%, p=0.003)$.

Our unadjusted and adjusted logistic regression models are shown in Fig. 1a, b, respectively. Variables significantly associated with mortality in our bivariate analyses at $\alpha=0.10$ level were included in our adjusted model: CKD, age group, sex, and hypertension. Specifically, the adjusted odds of dying were 1.51-fold (95\% CI $=1.19-1.90)$ higher for patients with CKD as compared to the non-CKD group. The adjusted odds of dying for the middle-aged and oldest age groups were 7.91-fold (95\% CI $=4.85-12.90)$ 
Table 2 Distribution of demographics, behavioral factors, and comorbidities by deceased status

\begin{tabular}{|c|c|c|c|c|}
\hline Variable & Sub-categories & $\begin{array}{l}\text { Non-deceased } \\
(n=6768,88.7 \%)\end{array}$ & $\begin{array}{l}\text { Deceased } \\
(n=856,11.2 \%)\end{array}$ & $p$ value \\
\hline \multirow[t]{3}{*}{ Age } & $<45$ years & $2075(99.1 \%)$ & $18(0.9 \%)$ & $<.001$ \\
\hline & 45 to $<65$ years & $2440(93.1 \%)$ & $181(6.9 \%)$ & \\
\hline & $65+$ years & $2253(77.4 \%)$ & $657(22.6 \%)$ & \\
\hline \multirow[t]{2}{*}{$\operatorname{Sex}^{\mathrm{a}}$} & Female & $3099(89.8 \%)$ & $351(10.2 \%)$ & 0.011 \\
\hline & Male & $3656(87.9 \%)$ & $505(12.1 \%)$ & \\
\hline \multirow[t]{5}{*}{ Race/ethnicity } & African ancestry & $1672(88.1 \%)$ & $226(11.9 \%)$ & 0.054 \\
\hline & White & $1654(87.3 \%)$ & $240(12.7 \%)$ & \\
\hline & Hispanic/Latinx & $1673(89.8 \%)$ & $189(10.1 \%)$ & \\
\hline & Asian & $318(89.1 \%)$ & $39(10.9 \%)$ & \\
\hline & Other/unknown & $1451(90.0 \%)$ & $162(10.0 \%)$ & \\
\hline \multirow[t]{3}{*}{ Smoking status } & Non-smoker & $3499(89.6 \%)$ & $407(10.4 \%)$ & $<0.001$ \\
\hline & Current/former smoker & $1307(83.9 \%)$ & $251(16.1 \%)$ & \\
\hline & Unknown & $1962(90.8 \%)$ & $198(9.2 \%)$ & \\
\hline \multirow[t]{2}{*}{ Asthma } & No & $6466(88.7 \%)$ & $823(11.3 \%)$ & 0.41 \\
\hline & Yes & $302(90.1 \%)$ & $33(9.9 \%)$ & \\
\hline \multirow[t]{2}{*}{ COPD } & No & $6617(89.1 \%)$ & $809(10.9 \%)$ & $<0.001$ \\
\hline & Yes & $151(76.3 \%)$ & $47(23.7 \%)$ & \\
\hline \multirow[t]{2}{*}{ HTN } & No & $5239(91.3 \%)$ & $498(8.7 \%)$ & $<0.001$ \\
\hline & Yes & $1529(81.0 \%)$ & $358(19.0 \%)$ & \\
\hline \multirow[t]{2}{*}{ Obesity } & No & $6322(88.9 \%)$ & $792(11.1 \%)$ & 0.33 \\
\hline & Yes & $446(87.4 \%)$ & $64(12.6 \%)$ & \\
\hline \multirow[t]{2}{*}{ Diabetes } & No & $5666(90.2 \%)$ & $614(9.8 \%)$ & $<0.001$ \\
\hline & Yes & $1102(82.0 \%)$ & $242(18.0 \%)$ & \\
\hline \multirow[t]{2}{*}{ HIV } & No & $6657(88.7 \%)$ & $846(11.3 \%)$ & 0.30 \\
\hline & Yes & $111(91.7 \%)$ & $10(8.3 \%)$ & \\
\hline \multirow[t]{2}{*}{ Cancer } & No & $6358(89.1 \%)$ & $782(10.9 \%)$ & 0.003 \\
\hline & Yes & $410(84.7 \%)$ & $74(15.3 \%)$ & \\
\hline \multirow[t]{2}{*}{ Chronic Kidney Disease } & No & $6309(89.8 \%)$ & $718(10.2 \%)$ & $<0.001$ \\
\hline & Yes & $459(76.9 \%)$ & $138(23.1 \%)$ & \\
\hline
\end{tabular}

Data were summarized as median (IQR) for continuous variables and as frequency (row \%) for categorical variables

${ }^{\mathrm{a}}$ Missing information on sex for $13(0.17 \%)$ patients and 29.51-fold (95\% CI $=18.32-47.53$ ) higher than that observed among the youngest age group. Males had 1.33fold (95\% CI $=1.14-1.55)$ higher adjusted odds of dying. Additionally, the adjusted odds of dying were 1.23-fold (95\% CI $=1.01-1.48)$ higher for patients with hypertension as compared to those without hypertension. Race/ethnicity, smoking status, COPD, diabetes, and cancer were not associated with mortality in our model.

\section{Discussion}

During the COVID-19 pandemic, social distancing and avoiding medical facilities is not a viable option for millions of individuals living with CKD. Due to their weakened immune system, CKD patients are more likely to experience adverse outcomes compared to the general population. Emerging evidence indicates associations between severity of COVID-19 and comorbidities like hypertension, diabetes, COPD, obesity, chronic lung disease, and CKD [10, 15]. In this investigation, we used a recent case series of patients diagnosed with COVID-19 across the MSHS from March 28th, 2020 to April 16th, 2020 to evaluate the impact of CKD on COVID-19-related mortality. We found a significantly increased risk of mortality among patients with CKD, independent of other patient characteristics. Overall, the attributable risk of COVID-19-related mortality due to CKD was approximately 0.128 , implying that $1 / 8$ deaths were due to CKD.

Despite the paucity of available studies, findings in another study culled these results by reporting that COVID19 patients with CKD were more likely to be on ventilators, 


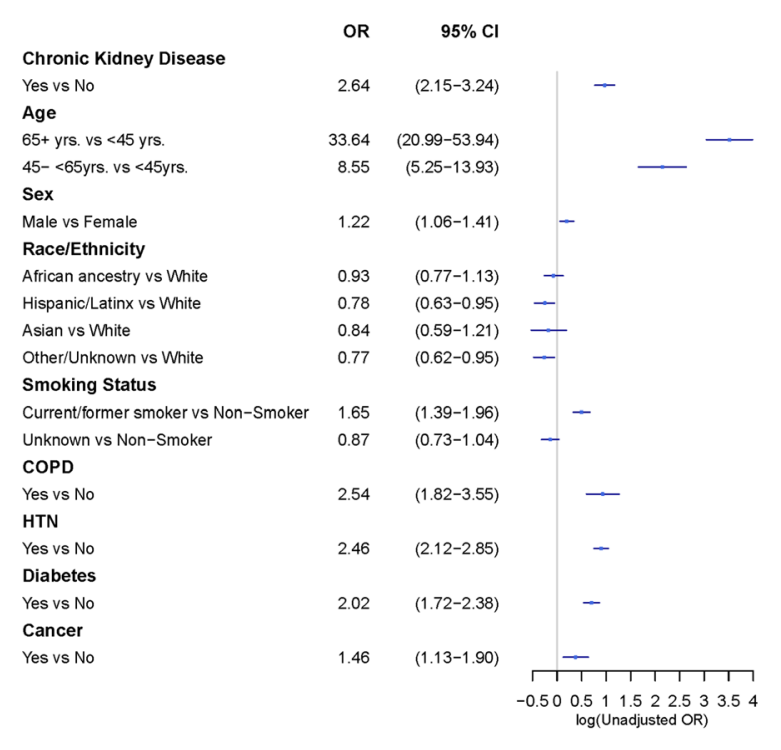

(a)

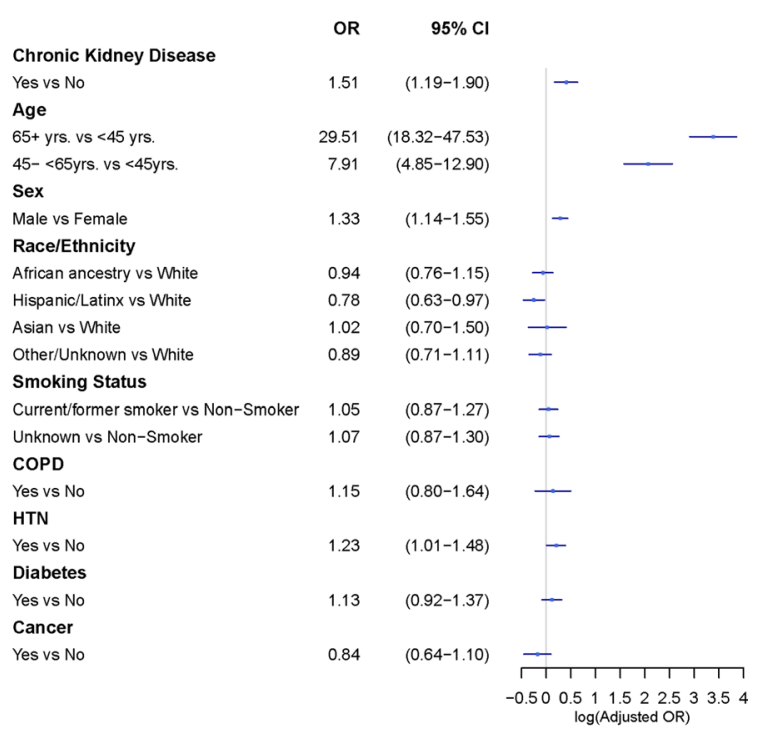

(b)
Fig. 1 Results of unadjusted (a) and adjusted (b) logistic regression analyses examining the contribution of CKD, as well as demographics, behavioral factors, and comorbidities to the odds of mortality

be admitted to the Intensive Care Unit (ICU), and have greater mortality rates [10]. In comparison to our study, a similar study conducted on a European population showed that in-hospital deaths were higher in patients with increased creatinine levels and history of previous CKD [16]. Similarly, other individual studies considered in a meta-analysis showed no association between CKD and severe COVID-19 disease; however, the pooled estimate of these studies indicated that patients with CKD had a significantly higher risk of severe diseases [17]. A possible explanation is that 3 out of 4 of the studies discussed in the meta-analysis may have been underpowered to detect any significant difference given that their sample sizes were 12, 138, and 140. Additionally, these studies include patients from different countries, where COVID-19 hospital admissions criteria may have differed from the criteria of our cohort in the US, thus allowing data on CKD status of COVID-19 patients to be lost due to underreporting of comorbidities of patients treated at homes. This might explain the variations in COVID-19-CKD associations observed in different studies. Furthermore, recent clinical evidence has shown that non-survivors of COVID19 had elevated levels of factors related to kidney diseases such as creatinine, blood urea, neutrophil count, and D-dimer $[18,19]$. Additionally, in terms of transmission and pathology, COVID-19 uses the ACE2 receptor for initial viral attachment and human-to-human and cross-species transmission. A preponderance of which are located in the lungs and kidney-hence COVID-19's potential relationship with CKD [20]. Consequently, ACE inhibitors, often prescribed among COVID-19-positive patients. Variables were included in this analysis if they were associated with mortality at the $\alpha=0: 10$ level in bivariate assessments

for CKD comorbidities, may leave users at increased risk for adverse COVID-19 symptomology and outcome [21, 22].

We observed patients who had COVID-19 and CKD had other coexisting morbidities such as diabetes, hypertension, and COPD, and increased health risk behavior. We found a significant association with old age, male gender, and hypertension as predictors of mortality within our cohort, while accounting for the presence of additional comorbidities, and risk behavior, which might explain intrinsic susceptibility to becoming critically ill upon infection. Support for our findings stems from previous studies, reporting that older patients were more likely to die from COVID-19 [23, 24]. A study identified that patients with COVID-19 were over 63 years of age on average, more likely to be male and have an additional comorbidity, including hypertension [10]. However, in contrast to other published work, our study found no relationship with other comorbid conditions, despite the variability and diversity in the population with respect to race and CKD status. Additionally, a meta-analysis on over 1500 patients found that old age and comorbidities such as hypertension, diabetes, respiratory system disease, and cardiovascular disease are risk factors for seriously ill COVID-19 patients [25]. A potential explanation for this discrepancy is patients in our study could have had better pre-admission control of other comorbid conditions. Additionally, our patient cohort is more heterogeneous regarding chronological age, which may have resulted in low rates of reported comorbidities typically associated with older age in this study. 
Studies have shown that African Americans (AA) diagnosed with CKD have a significantly higher risk of mortality than white patients with CKD, and evidence suggests a link between socioeconomic factors and worse CKD outcomes [9]. While white individuals had a greater mortality rate than other races in our study $(12.7 \%)$, controlling for other variables, the difference was not statistically significant. Considering that most deaths reported are based on in-hospital reports, it is possible that more white individuals are accounted for due to their better access to healthcare, whereas minorities are more likely to constitute the out-of-hospital COVID-19 mortality and their deaths are unaccounted [26]. Evidence also suggests that CKD outcomes do not improve in racial minorities when care is standardized [9].

Our results confirmed shared COVD-19 and CKD demographic and clinical characteristics documented in the CKD health disparities in the US (e.g., older age, comorbidities). To reduce the effect of COVID-19 on existing CKD disparities and to improve the health of vulnerable patients, healthcare systems must optimize CKD clinical care and reduce risk of infection and worse outcomes of COVID-19. A recent COVID-19 paper argued that parameters for the prediction of the need for admission to ICUs are urgently needed for patients with nephritis to enable timely management and appropriate resource allocation [27]. Routine data collection of differential clinical (morbidity, mortality) and social determinants of health (socioeconomic factors, healthcare access, physical environment, individual and collective health behaviors) within electronic medical records and health equity surveillance systems are necessary to optimize understanding of CKD-COVID-19 double burden [28, 29]. The surveillance system would benefit from local knowledge and active involvement of clinical supportive care staff (e.g., social workers) to facilitate understanding of broader contextual factors that can drive mortality and morbidity associated with outbreaks including COVID-19 [28-30].

Our study presents important findings. With a large sample size, of over 7000 patients from the epicenter of the pandemic in the US, our study is reflective of a broad patient demographic and outcomes in New York City. Our study does have limitations Data on clinical and behavior characteristics of patients, such as race and smoker status were missing. Additionally, information on patients that were on dialysis or that have had kidney transplants was not available. Future studies should investigate the impacts of these factors on outcomes of patients with COVID-19 and CKD. The laboratory data are not fully linked to the dataset, and will require analysis in a future study. Another limitation is that our study does not account for cases and deaths outside of the MSHS system, such as patients in nursing homes, homebound, and other care facilities.
In conclusion, the present study demonstrates that CKD is a strong predictor of mortality in patients diagnosed with COVID-19. Specifically, the odds of mortality are 1.51 times higher in CKD patients with COVID-19 when controlling for other risk factors. Our results indicate the importance of prioritizing COVID-19 patients with CKD in hospital admissions and guide closer monitoring and supportive care. Longitudinal studies are necessary to further explore the impact of COVID-19 on long-term kidney function, survival, and quality of life.

Author contributions NEM, KEO, NK, and KKB: protocol/project development and manuscript writing/editing. EKTB: protocol/project development, data collection or management, and data analysis. VA: data analysis. TGK, WN, AR, OAI, HF, MM, and KNM: manuscript writing/editing.

Funding None.

Availability of data and material (data transparency) https://msdw. mountsinai.org/.

Code availability (software application or custom code) SAS 9.4 and R 3.6.

\section{Compliance with ethical standards}

Conflict of interest The authors declare that they have no conflict of interest.

Ethical approval Waiver of documentation of informed consent by Ethics Committee of MSHS.

\section{References}

1. World Health Organization (2020) Coronavirus Disease (COVID19) - events as they happen World Health Organization. https:// www.who.int/emergencies/diseases/novel-coronavirus-2019/event s-as-they-happen

2. ArcGIS Dashboards (2020) Coronavirus Johns Hopkins dashboard link-DEV. https://dev.to/tonetheman/coronavirus-johns-hopkinsdashboard-link-73e

3. Duddu P, Praveen (2020) Verdict media limited. Pharmaceutical Technology. https://www.pharmaceutical-technology.com/featu res/covid-19-coronavirus-top-ten-most-affected-countries/

4. WPIX (2020) Latest coronavirus updates in New York https:// www.pix11.com/news/coronavirus/latest-coronavirus-updates-innew-york-thursday-april-23-2020

5. Reals T (2020) Coronavirus updates from April 22, 2020. CBS News, CBS Interactive https://www.cbsnews.com/live-updates/ coronavirus-update-covid-19-2020-04-22/

6. Chinese CDC Weekly (2020) The epidemiological characteristics of an outbreak of 2019 novel coronavirus diseases (COVID- 19): The novel coronavirus pneumonia emergency response epidemiology team https://cdn.onb.it/2020/03/COVID-19.pdf.pdf

7. Roser M, Ritchie H, Oritz-Ospina E, Hasell J (2020) Coronavirus Disease (COVID-19). [Internet] Our World in Data. https://ourwo rldindata.org/coronavirus 
8. Lee WC, Lee YT, Li LC, Ng HY, Kuo WH, Lin PT et al (2018) The number of comorbidities predicts renal outcomes in patients with stage 3-5 chronic kidney disease. J Clin Med 7:493

9. Norris K, Nissenson AR (2008) Race, gender, and socioeconomic disparities in CKD in the United States. JASN 19:1261-1270

10. Guan WJ, Liang WH, Zhao Y et al (2020) China medical treatment expert group for Covid-19 comorbidity and its impact on 1590 patients with Covid-19 in China: a nationwide analysis. Eur Resp J 55(5):200054

11. Mountsinai.org. 2020 [online] https://www.mountsinai.org/files /MSHealth/Assets/HS/About/Coronavirus/MSHS-TreatmentGuidelines-COVID.pdf

12. Roche Molecular Systems, Inc (2020) cobas SARS-CoV-2: Qualitative assay for use on the cobas ${ }^{\circledR}$ 6800/8800 Systems. Roche Molecular Systems, Inc., New Jersey

13. Levey AS, Bosch JP, Lewis JB, Greene T, Rogers N, Roth D (1999) A more accurate method to estimate glomerular filtration rate from serum creatinine: a new prediction equation. Modification of Diet in Renal Disease Study Group. Ann Intern Med 130:461-470

14. Levey AS, Coresh J, Balk E et al (2003) National kidney foundation practice guidelines for chronic kidney disease: evaluation, classification, and stratification. Ann Intern Med 139:137

15. Dietz W, Santos-Burgoa C (2020) Obesity and its implications for COVID-19 mortality. Obesity 28(6):1005

16. Portolés J, Marques M, López-Sánchez $\mathrm{P}$, de Valdenebro M, Muñez E, Serrano ML, Malo R, García E, Cuervas V (2020) Chronic kidney disease and acute kidney injury in the COVID19 Spanish outbreak. Nephrol Dial Transplant 35(8):1353-1361. https://doi.org/10.1093/ndt/gfaa189

17. Henry BM, Lippi G (2020) Chronic kidney disease is associated with severe coronavirus disease 2019 (COVID-19) infection. Int Urol Nephrol 52(6):1193-1194

18. Wang D, Hu B, Hu C, Zhu F, Liu X, Zhang J et al (2020) Clinical characteristics of 138 hospitalized patients with 2019 novel coronavirus-infected pneumonia in Wuhan. China Jama 323(11):1061

19. Li Z, Wu M, Yao J, Gu J, Liao X, Song S et al (2020) Caution on kidney dysfunctions of COVID-19 patients. MedRxiv. https://doi. org/10.2139/ssrn.3559601

20. Wan Y, Shang J, Graham R, Baric RS, Li F (2020) Receptor recognition by the novel coronavirus from Wuhan: an analysis based on decade-long structural studies of SARS coronavirus. J Virol. https://doi.org/10.1128/JVI.00127-20

21. Zheng YY, Ma YT, Zhang JY, Xie X (2020) COVID-19 and the cardiovascular system. Nat Rev Cardiol 17(5):259-260

22. Fang L, Karakiulakis G, Roth M (2020) Are patients with hypertension and diabetes mellitus at increased risk for COVID-19 infection? Lancet Resp Med 8(4):e21

23. Zhou F, Yu T, Du R, Gouhui F, Liu Y, Liu Z et al (2020) Clinical course and risk factors for mortality of adult patients with COVID-19 in Wuhan, China. Lancet 395(10229):1054-1062

24. Deng Y, Liu W, Liu K, Fang YY, Shang J, Ling Z et al (2020) Clinical characteristics of fatal and recovered cases of coronavirus disease 2019 (COVID-19) in Wuhan, China: a retrospective study. Chin Med J 133(11):1261-1267

25. Yang J, Zheng Y, Gou X, Pu K, Chen Z, Gua Q et al (2020) Prevalence of comorbidities and its effects in patients infected with SARS-CoV-2: a systematic review and meta-analysis. Int J Infect Dis 94:91-95

26. Sohn H (2017) Racial and ethnic disparities in health insurance coverage: dynamics of gaining and losing coverage over the life-course. Popul Res Policy Rev 36(2):181-201. https://doi. org/10.1007/s11113-016-9416-y

27. Gross O, Moerer O, Weber M, Huber T, Scheithauer S (2020) COVID-19-associated nephritis: early warning for disease severity and complications. Lancet 20:31041-31042

28. Fielding JE, Kumanyika S, Manderscheid RW (2013) A Perspective on the development of the healthy people 2020 framework for improving U.S. population health. Public Health Rev 35(1):3

29. Wang Z, Tang K (2020) Combating COVID-19: health equity matters. Nat Med 26:458. https://doi.org/10.1038/s41591-020-0823-6

30. Gottlieb S, Rivers C, McClellan MB, Silvis L, Watson C (2020) National coronavirus response: a roadmap to reopening. American Enterprise Institute. https://www.aei.org/research-products/repor t/national-coronavirus-response-a-road-map-to-reopening/

Publisher's Note Springer Nature remains neutral with regard to jurisdictional claims in published maps and institutional affiliations. 